\title{
Experiencias resonantes de comunicación en pueblos indigenas de Oaxaca, México ${ }^{1}$
}

\author{
Carlos Baca-Feldman ${ }^{2}$ \\ Universidad Autónoma de Puebla, México ${ }^{3}$ \\ bacafeldman@gmail.com \\ Recibido: 19 de enero de 2015 \\ Aceptado: 11 de mayo de 2015 \\ Disponible en línea: 30 de noviembre de 2015
}

\footnotetext{
1 El artículo de reflexión que aquí se presenta tiene como objetivo vislumbrar, desde la teoría crítica, la manera en que el intercambio de experiencias que se da en los medios indígenas en Oaxaca, México, se teje como posibilidad y contradicción de la creación de una comunicación que vaya más allá de los parámetros capitalistas. El proyecto se enmarca dentro del Programa de Becas Nacionales para estudios de posgrado del Consejo Nacional de Ciencia y Tecnología (CONACYT) en México.

2 Maestría en Sociología y Licenciatura en Ciencias de la Comunicación.

3 Estudiante de Doctorado en Sociología, Instituto de Ciencias Sociales y Humanidades "Alfonso Vélez Pliego".
} 


\title{
Experiencias resonantes de comunicación en pueblos indigenas de Oaxaca, México
}

\section{Resumen}

En este texto quiero tomar la perspectiva de una lucha que intenta configurarse como experiencia de enfrentamiento a la destrucción que trae consigo la penetración del capital, que se ve reflejada en la utilización de las Tecnologias de la Información y Comunicación (TICs) como rebelión por parte de los pueblos indígenas en Oaxaca, México. Para ello, y tomando en cuenta diversos abordajes de la teoría crítica y los estudios de la comunicación, estudio el proceso de generación colectiva de mensajes como un espacio de creación de experiencias históricas de lucha, que tienen como objetivo común la construcción de prácticas emancipatorias que puedan engendrar 'otra comunicación'. La negatividad, la colectividad y la historia son las líneas que nos permiten pensar este tipo de experiencias, centrando la mirada, en todo momento, en las posibilidades, contradicciones y límites que les enmarcan.

Palabras clave: experiencias resonantes de comunicación; negatividad; colectividad; historia; apropiación tecnológica

\section{Resonant communication experiences of indigenous Oaxaca peoples, Mexico}

\begin{abstract}
In this text I want to take the perspective of a struggle that tries to shape itself as an experience of confrontation of the destruction originated by the penetration of capital. This is reflected in the use of Information Technology and Communication (ICT) as a rebellion by indigenous peoples in Oaxaca, Mexico. To that end, and taking into account various approaches to critical theory and communication studies, the process of collective message generation is studied as a space for creation of historical experiences of struggle with the common goal of building emancipatory practices which could generate 'another kind of communication'. Negativity, collectivity and history are lines that allow us to approach this kind of experiences, focusing our view at all times on the possibilities, contradictions and limits that frame them.
\end{abstract}

Keywords: resonant communication experiences; negativity; collectivity; history; technological appropriation.

\section{Experiências ressonantes de comunicação em povos indigenas de Oaxaca, México}

\begin{abstract}
Resumo
Neste texto quero tirar a perspectiva de uma luta tentando se configurar como experiência de defrontação à destruição que consigo traz a penetração do capital, que reflete na utilização das Tecnologias da Informação e Comunicação (TICs) como forma de rebelião dos povos indígenas de Oaxaca, México. Para isso, e levando em conta diversas abordagens da teoria crítica e os estudos da comunicação, estuda-se o processo de geração coletiva de mensagens como espaço de criação de experiências históricas de luta, que tem como objetivo comum a construção de práticas emancipatórias que poderiam engendrar uma 'outra comunicação'. A negatividade, a coletividade e a história são as linhas que permitem nós pensarmos este tipo de experiências, focando o olhar, em todo momento, nas possibilidades, contradições e limites que as enquadram.
\end{abstract}

Palavras-chave: experiências ressonantes de comunicação; negatividade; coletividade; historia; apropriação tecnológica 
A partir de los años noventa los mecanismos de penetración del capital en las esferas de la vida han incrementado y se han hecho más agresivos, sobre todo con la implementación de las políticas neoliberales en gran parte del mundo (Harvey, 2007). En este mismo periodo, la incursión de nuevas Tecnologías de la Información y Comunicación (TICs) en sectores de la población que antes no tenían acceso a ellas (como Internet, las cámaras de video caseras, los transmisores de radio, etc.) ha permitido que diferentes grupos sociales se adueñen de estas tecnologías para poder transmitir el punto de vista propio de su modo de entender(se) (en) el mundo y hacer frente a la información vertical que se transmite en las empresas de comunicación. Por ello mismo, las experiencias de comunicación no son elementos aislados de las luchas; pertenecen a ellas y pelean junto y para ellas. En palabras de John Holloway,

El teatro, la poesía y el humor [yo añadiría a la utilización de los medios de comunicación] han sido un elemento clave en el impacto de los zapatistas y otros movimientos: no como instrumentos del movimiento, sino como elemento central del movimiento mismo. (2011, p. 113)

La diversidad de herramientas comunicacionales existentes en la actualidad es tan extensa y sus procesos de realización tan diferentes que sería una tarea interminable tratar de pensar todas estas en el entramado de las luchas sociales. Pero tampoco resulta adecuado, desde mi propio criterio, descartar el hecho de que se vive un momento de convergencia tecnológica; ninguna de las tecnologías de la comunicación es utilizada por sí sola, sino que convive con otras herramientas. Los pueblos indígenas no escapan a esta lógica y se han apropiado de las TICs para denunciar diferentes atropellos a sus formas de vida, para preservar sus tradiciones y su lengua e informar sobre asuntos que conciernen a la propia comunidad, entre otras. La intención de este texto es pensar cómo la apropiación de las tecnologías y los mecanismos de expresión por parte de los pueblos indígenas pueden comprenderse como experiencias resonantes de comunicación que expresan las posibilidades, contradicciones y límites de las luchas anti-capitalistas. 
En este orden de ideas, el estado de Oaxaca brinda las condiciones que se requieren para lograr este objetivo. Por un lado, las condiciones topológicas del estado han posibilitado que muchas de las comunidades se mantuvieran 'aisladas' de los medios masivos de comunicación por mucho tiempo. Por otro lado, en parte por esta misma condición, muchos de los programas de expansión de las formas capitalistas, tanto a nivel económico como político y social, no han penetrado del todo en muchos espacios. Estas condiciones, aunque no solamente estas, han facilitado la creación de muchas experiencias resonantes de comunicación, que van desde radios comunitarias, pasando por colectivos de video, hasta llegar a las más avanzadas, como las redes comunitarias de telefonía celular e Internet.

Lo que se presenta en el estado de Oaxaca no es la utilización de medios y su proliferación como simples procesos comunicativos, sino que sirven a las luchas y resistencias que históricamente se han venido generando en esta región. Por poner algunos ejemplos: la historia de Estéreo comunal nos llevaría hasta la venta de los bosques en Guelatao; los antecedentes de Radio totopo tienen que ver con la colocación de ventiladores gigantes para obtener energía eólica en el Itsmo; el surgimiento de Ojo de Agua Comunicación está relacionado con la idea del multiculturalismo pero también con el levantamiento zapatista; la Red de Telefonía Comunitaria en Talea de Castro no solo se refiere al deseo de comunicarse a través de los aparatos celulares, sino también a la necesidad de hacer frente a los monopolios de las telecomunicaciones; las radios en la costa de las comunidades afrodescendientes están ligadas a procesos de exclusión racial históricos y Radio Plantón a los problemas que enfrentan los maestros, pero también a la necesidad de articular el descontento social en un movimiento masivo como la APPO.

La lista podría continuar, pero lo importante es ver cómo diferentes procesos de penetración del capital han generado luchas que impiden que estos procesos se desarrollen del todo, y cómo dichas luchas se apoyan en herramientas de la comunicación para hacerse resonar. Pero ello no es un proceso sencillo; requiere ver en él una serie de elementos que, aunque contradictorios, también contienen las posibilidades de construcción de 'otro mundo posible'. Considero que la manera más 
adecuada de hacerlo es poniendo en el centro a los sujetos hacedores y receptores de la comunicación.

Mi intención no será presentar un mapa de articulaciones de las luchas sociales y la utilización de las tecnologías de la comunicación a favor de ellas, sino ver los diferentes mecanismos de apropiación que utilizan los sujetos para alcanzar sus propios ideales a través del intercambio de experiencias, aunque estos puedan resultar contradictorios. Para lograr este objetivo, comenzaré por una discusión de las características de la comunicación en el capitalismo y las luchas que se dan en torno a ella.

\section{Contradicciones y posibilidades de la comunicación en el proceso de totalización capitalista}

Si pensamos desde una perspectiva que centre la mirada en las relaciones sociales como la manera de poder transformar nuestro contexto actual, nos damos cuenta que la forma en que nos organizamos económica, política y socialmente es contradictoria. En otras palabras, la manera en que nos relacionamos los unos con los otros en el modo de producción capitalista no está completamente dada o condicionada por las categorías, normas y formas de comprender el mundo propuestas como formas naturales de comportarnos (Marx, 2010). Por lo tanto, para poder pensar en la construcción de distintas relaciones sociales es necesario que comprendamos que no somos sujetos puros; no somos solo personas que rigen su actuar bajo el determinismo capitalista, pero tampoco estamos por completo alejados de este proceso totalizante.

Siguiendo a David Harvey (2007), la sociedad capitalista contemporánea se encuentra articulada a través de diferentes maneras en las que la información juega un papel clave en el equilibrio -siempre buscado pero rara vez alcanzado- del capital. En este sentido, la necesidad permanente de abrir nuevos espacios para la incursión de las formas capitalistas ha desencadenado una penetración constante en esferas de la vida y sujetos que antes no tenían, como medio de organización colectiva 
y producción, al capitalismo. Dada esta situación, los desarrollos tecnológicos que acompañan el continuo crecimiento del capital han sido reforzados por formas cada vez más complejas de comunicación, rompiendo barreras espaciales y temporales. Como señala Ignacio Ramonet, "el dominio del multimedia se convierte en un tema estratégico en los aspectos político, religioso, industrial y cultural" (1999, p. 222). Así las cosas, las TICs son elementos centrales del capital no solo porque permiten una mayor fluidez en la información que se requiere para hacer circular las mercancías, sino también porque son mercancías en sí mismas que representan un mercado cada vez más grande. Todo ello nos lleva a considerar la cada vez más certera la premisa de Guy Debord (1998), según la cual vivimos en la "sociedad del espectáculo".

Sin embargo, como elementos contradictorios, las TICs -al ser utilizadas por sujetos que luchan por encontrar nuevas maneras de comprender(se) (en) el mundo- van rompiendo formas fetichizadas de relacionarse entre sí, haciéndolas también herramientas que ayudan a la articulación de procesos colectivos de transformación social. Por ejemplo, Claudia Magallanes $(2005 ; 2008)$ al hablar del video indigena, nos señala las complejidades a las que se enfrentan los videastas para producir, distribuir y exhibir sus productos. En todo ese proceso, se enfrentan a lógicas del mercado con las que deben luchar para poder rescatar lo verdaderamente importante para ellos: la expresión de su propio punto de vista con la utilización de esas tecnologías mercantilizadas.

Sin embargo, no podemos pensar en los procesos sociales que se generan en tales circunstancias sin ver que la comunicación también se encuentra en una constante contradicción. Analizar este tipo de medios como procesos particulares significa verlos como elementos aislados y centrarnos únicamente en las potencialidades que se dan al interior de cada uno de ellos. Resulta necesario comprenderlos como parte de una sociedad que se encuentra en un proceso constante de totalización capitalista y que lucha por no dejarse penetrar del todo por ella. Como lo señala el antropólogo zapoteco Jaime Martínez Luna, 
Las contradicciones evidentes en la reproducción de la comunidad como tal, dentro del sistema global, resulta a todas luces una unidad que expresa una especificidad, no solo desde las premisas históricas que la sustentan, sino a través de su articulación actual al modo de producción capitalista en la que se reproduce, precisamente su esencia contradictoria. (2013, p. 158)

Siguiendo este argumento, resulta adecuado trasladar también el pensamiento de Adorno (2004) al estudio de las experiencias de comunicación de los pueblos indígenas, ello con el fin de escapar a las apariencias identitarias que se presentan, a primera vista, en los análisis. Por un lado, observamos los vínculos externos de la fetichización -de lo indígena o lo comunitario, por ejemplo-, en su relación con los medios masivos -la pobreza que les envuelve-. Por el otro, sus vínculos internos reflejan la formación de comunidad, la posibilidad de otra información y la comunicación como proceso de transformación social. Se trata de poder observar la doble dimensión que se esconde en estos procesos de comunicación para descubrir el antagonismo que les envuelve y los mantiene en constante movimiento de lucha. En este sentido, para superar la escisión sujeto-objeto que se produce en la comunicación bajo la supremacía capitalista, es necesario no entender el proceso comunicativo como un elemento aislado, sino como parte dialéctica negativa de la totalidad (Adorno, 1984).

Lo que resulta de ello es la potencialización de las posibilidades de articulación que se presentan en las experiencias de comunicación de los pueblos indígenas, sin dejar de lado las contradicciones y limites a los que se enfrentan. Una perspectiva así de compleja, como lo es nuestra vida en sociedad, puede aportar la apertura de espacios de reflexión que ayuden a potencializar las diferentes luchas anti-capitalistas en muchos espacios y no solo en las comunidades indígenas, a través de las herramientas de la comunicación. Para ello, desde mi punto de vista, un concepto que puede ayudarnos analíticamente es el de experiencias resonantes de comunicación. Veamos a qué me refiero. 


\section{¿Por qué experiencias resonantes de comunicación?}

La perspectiva del estudio de la comunicación que he venido narrando requiere poner en el centro a los sujetos; pensar en las personas que hacen y reciben mensajes de comunicación como elementos potencializadores de la misma. Es aquí donde considero que es adecuado (re) pensar las categorías que se nos han dado para el análisis de estos procesos sociales. La propuesta que traigo en este estudio es cambiar la categoria medios de comunicación por la de experiencias resonantes de comunicación. No creo que sea la única forma de nombrarlos, ni pretendo que así sea. Lo que me interesa recalcar a través de la discusión de esta categoría es que lo importante en aquellas experiencias que utilizan herramientas de la comunicación como elementos clave en su lucha política no son los mensajes en sí mismos, y tampoco los soportes que estos tengan, sino los sujetos hacedores y receptores de la comunicación en concordancia con las luchas más amplias que llevan a cabo.

Hablo de experiencias porque busco referirme a la constitución del sujeto. Si bien el sentido común de este término se refiere a los aprendizajes que permiten la 'adaptación' dentro de los procesos sociales, aquí me quiero referir a aquella forma de experimentar que constituye al sujeto, rompe con los procesos 'dados'y, en cierto sentido, está enmarcada por la inadecuación.

¿Por qué son resonantes estas experiencias? Entiendo la resonancia como la capacidad que tiene una determinada acción anti-capitalista de generar espacios de diálogo y lucha con otros actores sociales que comparten el deseo de transformación. Encuentro por lo menos cuatro maneras en las que la resonancia tiene lugar en este tipo de experiencias:

En primer lugar, la utilización de herramientas de la comunicación es un recurso que se nos presenta con una gran resonancia. Hoy, es posible dar a conocer sucesos muy lejanos geográficamente a través de las tecnologías de la comunicación. Gracias a ello, nos enteramos y podemos apoyar luchas que suceden en Grecia y los griegos pueden alzar 
su voz a favor de los problemas que tenemos en México. Muchos movimientos sociales han entendido muy bien este tipo de resonancia y, gracias a la utilización estratégica de las TICs, han logrado que mucha gente apoye su causa y se evite así el olvido de su lucha o, peor aún, la desaparición del movimiento o de las personas que colaboran con él. Tal es el caso de la lucha de las comunidades zapatistas en México, los Sin Tierra en Brasil y los piqueteros en Argentina, entre otros.

Cuando pensamos en ello, probablemente uno de los ejemplos que primero se nos viene a la mente es el de las comunidades zapatistas en Chiapas. Parece dificil pensar en la sobrevivencia de una lucha como la emprendida en estas comunidades sin la utilización de diferentes medios de comunicación (como el video, el Internet, la radio, la prensa, etc.), que les han permitido resonar a escala global. Probablemente por ello, estas comunidades son algunas de las que más materiales audiovisuales han producido.

Por otro lado, existen resonancias que posibilitan los soportes que adquiere la comunicación, al ser elementos que facilitan la memoria colectiva, haciendo de los mensajes una especie de archivos compartidos con generaciones futuras. Un ejemplo que se da frecuentemente es el retrato de las tradiciones y costumbres de los pueblos indígenas, hecho por ellos mismos y en su lengua propia. La intención al utilizar su propio lenguaje y dejar un testimonio hablado, por escrito o en un soporte audiovisual es que no se queden en el olvido las formas de organizar su vida, de relacionarse entre sí, con la naturaleza y con su espiritualidad. Pero este sentido de memoria a través de los soportes que facilita la comunicación no solo se da en los pueblos indígenas; también tenemos cada vez más registros que nos permiten acercarnos a muy distintos momentos de las luchas sociales.

En este orden de ideas, si pensamos en el empoderamiento de las audiencias en los 'medios ciudadanos', término que acuño de Clemencia Rodríguez (2009), podemos entender que una de las principales búsquedas es el rescate de su lengua tradicional. Es a través de la recuperación de la lengua como posibilitan la capacidad de 'nombrar' (Benjamin, 2003), pues no ven en el nuevo idioma que les ha sido 
impuesto (como el castellano en México) la relación entre las palabras y su manera de comprenderse en el mundo. Así, este tipo de experiencias pueden lograr los objetivos que Rodríguez propone. Las metas que deben guiar a los medios ciudadanos son: 1) fortalecer la identidad colectiva, 2) legitimar instituciones y responder a códigos sociales y 3) involucrar a la sociedad para facilitar las transformaciones.

Otro tipo de resonancia tiene lugar cuando la utilización de una cierta tecnología abre espacios para que otras también se utilicen. La historia de las experiencias de este tipo nos puede ayudar a aclarar esto. En diferentes comunidades donde se ha dado un uso constante de medios de comunicación, se han desencadenado colectivos que se han apropiado de otros soportes para hacer llegar sus mensajes. Este tipo de resonancias, conocidas como convergencia digital, no solo se dan al interior de las comunidades; también se transmiten las experiencias recibidas, con el paso del tiempo, a otros espacios y se generan distintas redes que permiten articular y potencializar los procesos locales de apropiación de las TICs.

A manera de ejemplos, en Santa Maria Tlahuitoltepec, hace ya varios años, se creó Radio Jënpoj, y posteriormente se iniciaron otros proyectos como la Licenciatura en Comunicación Comunal de la UNICEM. De la misma manera han surgido colectivos artísticos y mediáticos, y encuentros como la $2^{\circ}$ Cumbre Continental de Comunicadores Indígenas del Abla Yala, en 2013. Otro ejemplo más es el de Guelatao, donde una comunidad de menos de 1000 personas cuenta con dos radios -una semi-estatal (La voz de la Sierra Juárez) ${ }^{4}$ y otra comunitaria (Estéreo comunal)- y una televisora propia, de lo que surgió un proyecto que ha impactado a diferentes comunidades en el estado conocido como Campamento Audiovisual Itinerante. Un tercer ejemplo es el proyecto más innovador hasta el momento, inclusive a nivel internacional: la red comunitaria de telefonía celular desarrollada en Talea de Castro. Presento estos casos solo por poner unos ejemplos, pero la realidad es que esta situación se repite en muchas comunidades del estado y en la capital misma.

4 Perteneciente al Sistema de radiodifusoras indigenistas de la Comisión nacional para el desarrollo de los pueblos indigenas. 
Por último, la resonancia no solo se da en una vía -de los comunicadores a las audiencias-; también es un proceso en el que la generación de contenidos afecta a los propios comunicadores en la creación de los mismos y en la forma de abordarlos. A su vez, ellos afectan otros espacios y a las mismas luchas con sus productos, dando lugar, así, a una constelación de resonancias. En otras palabras, al hacer los mensajes, los comunicadores se vuelven parte de las luchas que desean retratar y, a su vez, influyen en la lucha misma, no solo a través de sus mensajes mediáticos sino como sujetos activos dentro de ellas.

En este sentido, un aspecto que también resulta relevante es el hecho de que se han generado diversas redes y espacios de diálogo que generan, a su vez, resonancias entre los propios comunicadores en Oaxaca. Por un lado, hay reuniones constantes entre comunicadores del estado para intercambiar sus experiencias y promover proyectos colectivos; por el otro, hay distintas organizaciones que funcionan como apoyo para que las comunidades generen sus propias experiencias resonantes. Algunas de ellas son Ojo de Agua Comunicación, Redes A.C., Palabra Radio y el Consejo Estatal de Comunicación Indígena. Si bien estos espacios pueden potencializar los procesos particulares, también resulta necesario ver en qué sentidos apunta cada una de las redes que se forman y las organizaciones de apoyo, para encontrar si en realidad posibilitan procesos autónomos en la generación de mensajes o, más bien, homogeneizan los sentidos de la comunicación.

Por todo lo anterior, considero que los comunicadores, en su quehacer cotidiano, reflejan la generación de experiencias que se comparten en diferentes sentidos, no solo en sus vínculos internos o en los externos -a través del material que producen-, sino con una carga histórica de luchas y acontecimientos que se reflejan en su hacer. Por ello, así como no podemos separar a estas experiencias del proceso de totalización capitalista, tampoco es posible dividir las formas de resonancia que se dan en ellas.

Sin embargo, no se debe olvidar que existen diferentes contradicciones que envuelven a estos sujetos, inmersos en formas de dominación capitalista. Ejemplo de ello son los mecanismos de financiamiento, el 
antagonismo propio del trabajo concreto y la necesidad individual de sobrevivir en el capitalismo, la estética mercantilista heredada de los estándares académicos más difundidos, la separación de los sujetos que suele darse con el paso del tiempo y la difusión de los mensajes solo en espacios que ya apoyan a las luchas reflejadas en los mensajes, entre otros.

Por lo tanto, las experiencias resonantes de comunicación presentan posibilidades de creación y de expresar maneras alternativas de comprender(se) (en) el mundo, devolviéndoles su capacidad de intercambiar experiencias (Benjamin, 1998). De esta manera, el tipo de experiencia que quiero discutir en este texto es aquella que se teje colectiva, negativa e históricamente, como posibilidad y contradicción en, contra y más allá del capitalismo. Por lo anterior, no se trata de una experiencia individual, sino de lo que he querido nombrar experiencia resonante.

\section{Partir de la negación}

Tomando la perspectiva de la teoría crítica como centro de la propuesta que aquí presento, puedo decir que el punto de partida es la negación, reflejada en el quehacer de los comunicadores indígenas. En este orden de ideas, John Holloway dirá que, a partir de la negación, del deseo de querer romper, de gritar constantemente (2002), surge otro hacer; una actividad que no está marcada por el dinero ni por el poder (2011). Esta es la base de su teoría de la grieta como mecanismo de rompimiento con el esquema de relaciones sociales mediadas por la forma de la mercancía. En general, poner a las experiencias de comunicación indígena bajo esta perspectiva nos permite verlas más allá de su ontologización o su aplastamiento en la estructura del capital, y entenderlos como luchas antagónicas que reflejan el deseo de generar 'otra comunicación', más allá de su fetichización bajo el modo de producción capitalista.

Lo que se niega en estas experiencias resonantes se puede pensar en muy diversos sentidos. Aquí presento dos que considero fundamentales. Por un lado, lo que se pretende con estas experiencias es 
visibilizar a los sujetos de la comunidad que no encuentran cabida en los medios masivos de comunicación y dar a conocer las múltiples historias que no encuentran espacios de difusión. A través de ello, se rebelan contra el impedimento a ser partícipes del uso de tecnologías de la comunicación como formas de expresión de su propia experiencia individual y/o colectiva. Apoyando esta idea, Antoni Castells i Talens, al hablar del cine indígena, pone un énfasis especial en este hecho cuando señala que "Rompe estereotipos, incluidos los forjados por el resto del cine latinoamericano; se expresa en la lengua minorizada; y usa la ficción en forma subversiva" (2003 p. 2).

La contradicción que podemos encontrar en esta forma de expresión radica en el hecho de que la estética que se refleja en los materiales audiovisuales suele ser muy parecida a la que se presenta en los medios masivos. Esto se da, en primera instancia, por la propia formación de los comunicadores, que suele ser tomada de los conocimientos que brindan las escuelas formales de cine o comunicación, o por la capacitación por parte de 'expertos' en la creación mediática. En segunda instancia, esto suele darse también por la preferencia de las audiencias por los productos audiovisuales que pueden observar en los medios masivos. No debemos olvidar que las experiencias resonantes de comunicación indígena conviven con los medios comerciales y, muchas veces, son más vistos los segundos que los primeros, incluso por las comunidades mismas.

Ejemplo de esta contradicción es la experiencia que me contó Jonathan Zambrano (2011), videasta wayuu ${ }^{5}$. En una entrevista personal, Zambrano señalaba la complejidad de adaptar una historia a las necesidades del sistema de producción cultural venezolano, en contradicción con los deseos de la propia comunidad. Estas complicaciones iban en dos sentidos: en primer lugar, en el hecho de que se necesitó una serie de asambleas para definir la participación de la comunidad en su último filme, y en segundo, que debía desarrollar dos versiones: una para las comunidades indígenas -con escenas más largas-y otra para el público general -con un ritmo más rápido-. Por lo

5 El pueblo wayuu tiene su territorio en la frontera entre Venezuela y Colombia. Actualmente se encuentra luchando en contra de las mineras al aire libre que se han instalado en este espacio y también sufre por el conflicto que trae el contrabando de gasolina entre ambos países. 
tanto, el tiempo de elaboración de esta película de ficción fue de alrededor de cuatro años, en los que se trabajó no solo a partir de las ideas de un grupo específico de cineastas, sino de un diálogo continuo entre la comunidad, los actores, los productores, editores, etc.

Las comunidades indígenas, a través de las experiencias de comunicación que desarrollan, también desafian el hecho de que los mensajes transmitidos en los medios de comunicación tengan que ser mercancías. Por ejemplo, la mayor parte de los videos que se producen en estas experiencias no se rigen bajo la lógica de los copyrights o derechos de autor. Así pues, en el caso de las radios se procura que el fin de la misma no sea la obtención de ganancias a través de la publicidad, aunque las emisoras reciban recursos de otras formas para poder operar.

Sin embargo, esto nos lleva a una paradoja dificil de subsanar en las experiencias: si dedican su tiempo enteramente al trabajo comunicacional, sin tener un salario o una ganancia, no tienen la capacidad económica para satisfacer sus necesidades fundamentales y sostener el colectivo -como pueden ser la compra de computadoras o cámaras, el pago de la luz y la renta, etc.-. Así, la búsqueda de maneras de obtener recursos económicos es una constante. Algunas experiencias optan por restringir sus videos o vender espacios publicitarios y así obtener ganancias mediante la venta de estos. Por ejemplo, en Estéreo Comunal, en Guelatao, la radio se financia por medio de un programa de anuncios para los negocios del pueblo, a precios elegidos por los propios anunciantes, conocido como 'patrocinios solidarios'.

Algunos otros, como Ojo de Agua Comunicación, optan por gestionar recursos del Estado u organismos internacionales, lo cual puede llevar a una especie de mercantilización de los materiales que producen al tener que responder a las lógicas propias de la institucionalidad. Un tipo de financiamiento parecido, pero que responde a lógicas distintas de institucionalidad, surge cuando la asamblea del pueblo apoya la experiencia de comunicación. Ejemplo de ello es la obtención de recursos por parte de la Radio Comunitaria Yaxhil (Yaguila, Oaxaca). Arturo Guerrero, en entrevista personal (2011), relató lo siguiente: 
La autoridad les da recursos de lo que les llega, ellos son los que siguen comprando equipos, ellos son los que siguen pagando luz y todo. Y también los ciudadanos colaboran. A mí me tocó estar en su aniversario el año pasado y como se decidió en asamblea que se iba a hacer la fiesta a todos les tocó poner no sé qué tanto de tortillas. Esto [la radio] es del pueblo, no es un asunto de la radio.

Con base en los ejemplos anteriores podemos comprender que ver las contradicciones y resaltar aquello que se quiere negar facilita los espacios y tiempos para la construcción de otro tipo de relaciones sociales más allá de los límites propios del capitalismo. Negando la estructura de la comunicación como una plataforma que solo permite adecuaciones dentro de ella es como podemos construir quehaceres distintos que busquen romper con la escisión sujeto/objeto en el capitalismo. Tal es el potencial que, desde mi punto de vista, revisten con mayor fuerza las experiencias de comunicación de los pueblos indígenas. Pero la negatividad no es un proceso individual; por ello, la siguiente característica que encuentro en estas experiencias es la colectividad.

\section{Las experiencias son colectivas, no individuales}

Podriamos pensar que la importancia que tienen las experiencias resonantes es la capacidad de hacer que un determinado acontecimiento se pueda guardar en la memoria colectiva a través de un material mediático determinado. De esta forma se comparte una determinada experiencia subjetiva con otras personas y, cada vez que se observa el producto se puede volver a compartir. Aunque esto es en cierta medida real, no solo se trata de un comunicador retratando un acontecimiento mediante una forma de expresión como el lenguaje audiovisual, las emisiones radiofónicas o una página de Internet; la experiencia es más bien colectiva, pues se refleja tanto la intención del comunicador como la de cada uno de los miembros de la experiencia resonante de comunicación, entre ellos los participantes que cuentan la historia y, en ocasiones, la comunidad en su conjunto. Ello porque "el movimiento de las grietas es un movimiento de experiencia, muy a menudo un aprendizaje en lucha" (Holloway, 2011, p. 46). 
Un elemento sustancial en la forma de hacer comunicación que aquí se relata es el proceso de creación colectiva de un material mediático. A pesar de los diversos abordajes teóricos que han tratado de conceptualizar este tipo de medios alternativos, ciudadanos, comunitarios, radicales, etc., considero que una característica que los diferencia de los masivos, además de su adherencia a diversos tipos de lucha, es el hecho de que se presta más atención al proceso que al producto final (Gumucio, 2004; Rodríguez, 2009).

Para apoyar esta idea, Amalia Córdova (en Paz, 2004), refiriéndose al cine y video indígena, apoya la concepción colectiva en este tipo de experiencias. En respuesta a la diferencia entre el cine creado por los indígenas y el realizado por los antropólogos y sociólogos, señala:

Yo diría que es una mirada colectiva, es un pensamiento colectivo, pensar siempre en tu público y tu público está ahí mismo, es con quien tú estás grabando, es con quien tú estás trabajando, entonces es una mirada colectiva. Tú dejas de lado y a veces sacrificas la autoría y tu libertad creativa en pos de que el mensaje sea colectivizado, sea recibido mejor, sea emitido de una forma que no es personal necesariamente, tú eres un traductor de un pensamiento colectivo, el uso del plural, el nosotros y no el yo. (Córdova en Paz, 2006, s.p.)

Ejemplo de ello es que en la producción de video por parte de las comunidades indígenas -en mi conocimiento y, aunque no en todos, en muchos casos- las decisiones en torno a quién será parte del proyecto, cómo se realizará, cuál será el tema que se aborde, el público al que se quiere destinar y quiénes aparecerán en pantalla se toman en asamblea; es decir, la comunidad en su conjunto decide qué quiere y cómo lo va a realizar.

Me gustaría reseñar un ejemplo que me parece se adecua muy bien a esta idea. Se trata del trabajo de video-cartas realizado por el colectivo de video oaxaqueño Ojo de Agua Comunicación La idea de estos cortos documentales es dar la oportunidad de que los niños de las comunidades expresen a otros niños de otros pueblos la forma en la que viven; 
de esta manera, se da un proceso de constante retroalimentación y conocimiento entre comunidades geográficamente distanciadas. Lo que resulta interesante de estas experiencias no es solo el hecho de que los propios niños deciden los contenidos de la video-carta, sino el pensamiento y diálogo que se promueve durante la realización de la misma. Ideas similares se encuentran en los trabajos de la Escuela Audiovisual Infantil, en Colombia, y TV Semana, en Cuba.

Las experiencias y resonancias vienen, entonces, en dos sentidos: por un lado, dan y reciben conocimientos de otras formas de entender(se) (en) el mundo y, por el otro, configuran un espacio de reflexión crítica sobre sus propias maneras de vivir en comunidad. Asimismo, estos materiales y sus procesos de elaboración se encuentran enmarcados en la comunicación indígena; por lo tanto, juegan un papel importante en la lucha de estas comunidades que acompaña a la disputa por la conservación de la lengua propia, la defensa del territorio y el rescate de las tradiciones, al mismo tiempo que es un enfrentamiento a las formas clasificatorias y excluyentes que trae el capitalismo en su forma de penetrar en diferentes sociedades.

En otras palabras, el énfasis que la utilización de ciertas tecnologías de la comunicación con objetivos articuladores pone en las luchas sociales es, en primera instancia, el proceso antes que el producto. En las experiencias de comunicación que se encuentran ligadas a luchas sociales, el valor que se genera son las relaciones sociales y el conocimiento obtenido mediante la realización de un producto comunicacional, a diferencia de la importancia que se le atribuye, en los medios masivos, al producto terminado y puesto a la venta en el mercado. Así, lo que podemos ver y escuchar en estos medios puede no resultar atractivo en los estándares mediáticos introducidos por los conglomerados comerciales, pero cuando vemos más a fondo, nos dejan ver la importancia del de un proceso crítico en la elaboración de un determinado producto comunicacional. Apoyando esta concepción, Antoni Castells i Talens señala que "la videoproducción comunitaria puede contribuir al empoderamiento personal y colectivo, a la desmitificación de los medios comerciales, a revertir los roles de poder y a fortalecer la fuerza colectiva" (2003, p. 3). 
Por ello, partir del proceso significa ver la experiencia colectiva -en la que conviven presente, pasado y futuro, personal y socialmente- que se genera en el momento de la realización de un proyecto comunicacional ligado a espacios de lucha. Podemos ver un ejemplo de ello en la diferencia del tiempo de realización, el diálogo que se da entre los participantes, la apertura de ciertos espacios para la crítica directa de la audiencia, la búsqueda de lenguaje que aborde y exprese los objetivos mismos de la lucha; en general, estamos hablando aquí de la producción de sentido como forma de hacer resonar a las luchas sociales. Por lo tanto, podemos decir que el tercer elemento que caracteriza a este tipo de experiencias es que tienen un fuerte anclaje en la historia.

\section{Estas experiencias hacen resonar la historia}

Si bien hasta ahora me he concentrado en la negatividad y la colectividad como mecanismos de potencialización de la experiencia de los comunicadores indígenas, no es posible dejar de lado que la experiencia, desde este tipo de conceptualización, no es a-histórica. El interés de Benjamin (2009) por el ejercicio de narrar deriva en la importancia que este autor atribuye a la vivencia histórica de las experiencias colectivas como ruptura. Para Rosas, siguiendo a Benjamin, "Decir que la experiencia es lenguaje revela su transmisibilidad, su aprehensión a través de la memoria y su anclaje a la tradición" (1999, p. 175).

Si entendemos el tiempo histórico como un proceso de continuas rupturas y no como una línea constante hacia el 'progreso' de la sociedad, nos acercamos a una visión del mundo que presenta diversas perspectivas de transformación en las formas organizativas de la sociedad. Ello nos permite alejarnos de la visión evolucionista de la sociedad, en la que se piensa la historia como un camino en el que siempre se va hacia adelante, como lo proponen los paradigmas del progreso y del desarrollo (Benjamin, 2007). Considero necesario entender la historia más como una serie de luchas que generan articulaciones entre los actores sociales. 
Desde esta perspectiva, nos acercamos al concepto de ruptura del tiempo lineal homogéneo de Walter Benjamin (2007). Para él, resulta primordial ver la historia desde la lucha que se ha desarrollado en la construcción de objetos y sujetos en constante conflicto. En ese sentido, propuso una mirada que se centra en el antagonismo expresado en ciertos momentos en los que se rompe con la dinámica progresista del capitalismo, ofreciendo nuevos panoramas de visualización del quiebre o superación de la contradicción trabajo concreto/trabajo abstracto. Al mismo tiempo, estas rupturas no son momentos de un tiempo específico, sino que conviven en ellas el pasado, el presente y el futuro, expresando las esperanzas y límites de cada uno de ellos.

A manera de ejemplo, una ruptura que ha resultado clave en la forma en que se han desarrollado las experiencias de comunicación de los pueblos indígenas en México y América Latina se dio en 1994, con el levantamiento del Ejército Zapatista de Liberación Nacional (EZLN).

Con el levantamiento del EZLN en Chiapas, en enero de 1994, se dio un proceso de lucha que ha contribuido a debatir la forma en que el capitalismo ha provocado la exclusión y explotación de determinados grupos sociales, trayendo consigo la destrucción de culturas, tradiciones y formas de relacionarse entre los seres humanos y con la naturaleza, específicamente en relación con los pueblos indígenas. Este hecho significó -y significa aún- un proceso de toma de conciencia, por parte de los mismos pueblos y la sociedad en general, sobre la necesidad de otorgar espacios que incluyan estas formas de ver y pensar.

Por un lado, la mercantilización de la cultura indígena se ha encargado de presentarla como una visión 'mágica' a la que hay que acceder y vender, apoyada conceptualmente por teorías como el multiculturalismo. Por otro lado, los propios pueblos indígenas han comenzado a encontrar resonancia en otros pueblos que comparten su situación de lucha, la cual han desarrollado a lo largo del tiempo ante la opresión del capital. No son pocos los que comparten la idea, reforzada por estudios históricos, acerca de la importancia del levantamiento zapatista en la multiplicación de experiencias de comunicación de los pueblos indígenas en México, pero también en muchas otras partes del mundo. 
En pocas palabras, el levantamiento del EZLN, a diferencia de los proyectos estatales que se habían generado antes, ha logrado poner en la discusión pública el tema de los pueblos indígenas desde una visión que logra expresar su lucha histórica de más de 500 años, lo cual ha resultado en espacios de articulación de diferentes experiencias, caracterizadas por poner en el centro el conflicto de oscurecimiento y opresión que viven estos pueblos.

No podemos descartar de la historia de experiencias resonantes de comunicación de los pueblos indígenas en México los proyectos que se desarrollaron -sobre todo por el Estado mexicano- antes de los sucesos de 1994. Un ejemplo de ello son las iniciativas de las radios indigenistas y los centros de video indígena, surgidos a finales de los setenta y principios de los ochenta. Estas instituciones permitieron la capacitación de comunicadores indígenas, muchos de los cuales crearon después sus propias experiencias, mientras que otros permanecieron en estas instituciones del Estado (Ramos, 2006). Si bien estas instituciones tienden en algunos aspectos a verse como organismos públicos, también contienen articulaciones y formas de lucha debajo de las lógicas de información estatal (Castells, 2011).

Para finalizar este punto, traigo a colación un momento que, a mi parecer, ilustra bastante bien los momentos de ruptura del tiempo lineal homogéneo; a saber, el vivido en junio de 2006 en la ciudad de Oaxaca. Por un lado, el plantón de los maestros de la Sección 23 del Sindicato Nacional de los Trabajadores de la Educación (SNTE) y la creación del movimiento social conocido como la Asociación Popular de los Pueblos de Oaxaca (APPO), se valió de la utilización de diferentes medios de comunicación, que iban desde su propia estación radiofónica, Radio Plantón, hasta la toma de Radio Universidad, de emisoras comerciales y de la televisora del gobierno.

En el mismo periodo, el propio gobierno acudió a la utilización de un medio de comunicación no aprobado por la ley, al que se llamó Radio Ciudadana. Además de estos medios más convencionales, también se valieron del uso de herramientas de Internet. Por otro lado, en el verano de ese año, el colectivo Ojo de Agua Comunicación organizó 
el $6^{\circ}$ Festival de la Coordinadora Latinoamericana de Cine y Video de los Pueblos Indígenas, al que asistieron videastas de todo el mundo. Aunque los fines perseguidos por los integrantes de la APPO y los videastas indígenas parecían muy distantes entre sí, lo que sucedió fue que la ciudad se convirtió, por un momento, en un cúmulo de resonancias sobre las posibilidades que brindan distintas herramientas de la comunicación como potencializadoras de luchas sociales.

\section{A modo de conclusión}

La teoría crítica, a pesar de que parece contener un extrañamiento con respecto a la producción teórica de los pueblos indígenas, contiene una serie de elementos que nos permiten pensar la transformación social en el proceso de totalización capitalista. Resulta, desde mi punto de vista, muy importante voltear la mirada al trabajo de los comunicadores indígenas como parte de un proyecto más amplio, que ayuda a comprender de qué manera podemos apoyarnos en el uso de tecnologías de la comunicación en un sentido que vaya más allá del valor de cambio de una comunicación reificada. Como señala Martínez Luna, "La imagen para nosotros es ahora y siempre, al igual que la oralidad, la fórmula de la resistencia, de la reproducción de nuestros propios, en fin, de lo que somos y queremos en el futuro" (2013, p. 303).

Sin embargo, este proceso requiere de una mirada que nos permita ver las contradicciones que emergen de las formas de comunicación como prácticas emancipatorias, en conjunción con las posibilidades de pensar la construcción de 'otro mundo posible'. Para ello, a través de la ejemplificación de distintos procesos de comunicación en Oaxaca, México, la propuesta que he presentado se sustentó en la formulación del concepto de experiencias resonantes de comunicación, que tiene como elementos constitutivos la negatividad, la colectividad y la historia. 


\section{Referencias}

Adorno, T. (1984). Dialéctica negativa. Madrid: Taurus.

Adorno, T. (2004). Escritos sociológicos I. Madrid: Ediciones Akal S.A.

Benjamin, W. (1998). Expriencia y pobreza. En W. Benjamin, Discursos interrumpidos I (pp. 167-173). Madrid: Taurus.

Benjamin, W. (2003). Ensayos. Buenos Aires: Editorial Nacional Madrid.

Benjamin, W. (2007). Conceptos de filosofía de la historia. Buenos Aires: Terramar Ediciones.

Benjamin, W. (2009). Obras II. Madrid: Abada.

Castells i Talens, A. (2003). Cine indígena y resistencia cultural. Chasqui, 84, 50-57.

Castells i Talens, A. (2011). ¿Ni indigena ni comunitaria? La radio indigenista en tiempos neoindigenistas. Comunicación y sociedad, (15), 123-142.

Debord, G. (1998). La sociedad del espectáculo (Maldeojo Trad.). Archivo Situacionista Hispano. Recuperado de http://serbal.pntic.mec.es/ cmunoz11/Societe.pdf

Gumucio, A. (2004). El cuarto mosquetero. La comunicación para el cambio social. Revista Investigación y Desarrollo, 12(1), 2-23.

Harvey, D. (2007). Breve historia del Neoliberalismo. Madrid: Akal.

Holloway, J. (2002). Cambiar el mundo sin tomar el poder. Puebla: Insituto de Ciencias Sociales y Humanidades "Alfonso Vélez Pliego" - BUAP.

Holloway, J. (2011). Agrietar el capitalismo. Buenos Aires: Herramienta.

Magallanes, C. (2005). Video tecnología y medios independientes. Códigos, 1(2), 95-112. 
Magallanes, C. (2008). The use of video for political consciousness-raising in Mexico. An analysis of independent videos about the zapatistas. Nueva York: The Edwin Mellen Press.

Martínez Luna, J. (2013). Textos sobre el camino andado (Vol. I). Oaxaca: CSEIIO.

Marx, K. (2010). El Capital. Critica de la economía politica (Vol. I). México: Fondo de Cultura Económica.

Paz, M. (2006). Una mirada al video indígena latinoamericano. Entrevista con Amalia Córdova. Revista Antropología Visual, 5. Recuperado de http://www. antropologiavisual.cl/amalia_cordova.htm

Ramonet, I. (1999). Un mundo sin rumbo. Crisis del fin de siglo. Madrid: Editorial Debate S.A.

Ramos, J. M. (2006). La radio comunitaria en México: lecciones aprendidas y claves para el futuro. Coloquio internacional sobre medios comunitarios, participación, sostenibilidad e identidad (pp. 1-14). Santiago de los Caballeros, República Dominicana: Centro de Competencia en Comunicación para América Latina.

Rodríguez, C. (2009). De medios alternativos a medios ciudadanos: Trayectoria teórica de un término. Folios, (21-22), 13-25. Recuperado de http:/ / aprendeenlinea. udea.edu.co/revistas/index.php/folios/article/viewFile/6416/5898

Rosas, O. (1999). Walter Benjamin: Historia de la experiencia y experiencia de la historia. Argumentos, (35-36), 169-185.

\section{Cómo citar este artículo}

Baca-Feldman, C. (2016). Experiencias resonantes de comunicación en pueblos indigenas de Oaxaca, México. Universitas Humanística, 81, 255-277. http:// dx.doi.org/10.11144/Javeriana.uh81.ercp 